\title{
On Chip Implementation of a Pixel-Parallel Approach for Retinal Vessel Tree Extraction
}

\author{
C. Alonso-Montes \\ Dept. of Computer Science \\ Univ. A Coruña. SPAIN. \\ calonso@udc.es
}

\author{
P. Dudek \\ Dept. of Electrical and \\ Electronics Engineering \\ Univ. Manchester. UK \\ p.dudek@manchester.ac.uk
}

\author{
M. G. Penedo \\ Dept. of Computer Science \\ Univ. A Coruña. SPAIN \\ mgpenedo@udc.es
}

\begin{abstract}
Retinal vessel tree extraction from angiography images plays an important role not only in the medical domain, but also in biometric identification applications. From the image processing point of view, many algorithms and strategies have been developed to deal with this topic. Although reliable results have been obtained, the main disadvantage in most of these proposals is still the high computation effort required.

In this paper, a methodology to extract the retinal vessel tree has been developed, specially defined in terms of fine grain SIMD processing with the purpose of improving the computation time. The proposal has been implemented on a cellular processor array VLSI chip. The execution times for the main modules of the proposed algorithm have been included to show its capability.
\end{abstract}

\section{INTRODUCTION}

Currently, the computation of accurate geometric models of anatomic structures from medical images, like the retinal vessel tree, has become increasingly important in both authentication systems [1] and medical applications [2]. Although a lot of research effort has focused on developing algorithms and strategies for the retinal vessel extraction [3], less attention has been paid to the improvement of the computation time.

In this sense, the vessel extraction algorithm proposed in [4] intended to improve the execution time by means of developing all the steps under the Cellular Neural Network (CNN) [5] paradigm. This methodology finds the exterior of the vessels using an active contour technique, the so-called Pixel Level Snakes (PLS) [6]. Fitting the interior of the vessels has been the most usual approach to tackle the retinal vessel extraction [7], [8]. Nevertheless, the main disadvantage of these approaches is that they provide a limited control of the evolution process due to the tubular structures. Moreover, as it has been shown in [7], complex rules should be defined to avoid the contour flowing outside the vessel, whereas the initialisation is also more complex.

Our proposal is based on fitting the exterior of the vessels and contour evolution is then controlled in an easy way. The initialisation stage is also clearly easier, especially in an automatic process, since there is a larger proportion of background pixels. PLS have been implemented on hardware architectures with capabilities of single instruction multiple data (SIMD) processing, like the CNN-based chips [9] as well as the focal plane cellular processor array SCAMP-3 vision system [10], [11].
Although reliable results have been obtained in [4], some of the CNN-based steps for the estimation of the guiding information and the initial conditions for PLS, have been developed by means of non linear templates which prevents their implementation in the current generation of cellular processor VLSI chips such as those of either the ACEx family or the SCAMP-3.

In this paper, the original proposal addressed in [4] has been redefined in terms of local dynamic convolutions and morphological "hit and miss" operations together with arithmetic and logical operations to be implemented and tested in a fine grain SIMD processor array, particularly the SCAMP-3 chip [10]. The SCAMP-3 vision system executes a sequence of simple array instructions, like addition, inversion, oneneighbour access, operating in a pixel-parallel fashion on $128 \times 128$ arrays, at a rate of 1.25 MOPS per pixel.

The paper is structured as follows: in Section II the PLS performance is briefly described, Section III the proposed algorithm is explained, in Section IV the main results obtained with the SCAMP system are shown, and, finally in Section $V$ the main conclusions are discussed.

\section{Pixel LeVel Snakes (PLS)}

Pixel Level Snakes are a massively parallel active contour technique inspired by the energy-based deformable models. The inputs consist of a binary image containing the initial contours and a multi-bit image containing the guiding information, called external potential image, which guides the PLS evolution.

PLS contours evolve towards local minimal distance curves, based on a metric defined as a function of the features of interest. PLS algorithm operates along the four cardinal directions performing the evolution as a pixel-by-pixel shift (activation and deactivation of pixels in a binary image). The goal after each cycle (four iterations, one for each cardinal direction) is to obtain a new contour slightly shifted in order to be closer to the boundaries of interest. Like in conventional active contours, the evolution is controlled by means of the internal, the external and the balloon potentials. The internal potential controls the smoothing effect of the contour giving more robustness to the model against noise. The external potential guides PLS towards the boundaries of interest and 
it should be defined in such a way that the boundaries of interest coincide with the valleys of the potential field. The balloon potential controls the inflation or deflation tendency of a closed contour and it is usually required to guide the evolution when the external potential is too weak. Topological changes like merging and splitting contours are also easily handled by PLS. A comprehensive description of PLS can be found in [6].

In this paper, the PLS region-based approach proposed in [11] has been implemented, where the contours are implicity represented as the boundaries of active regions.

\section{Algorithm}

The methodology proposed in this paper (Fig. 1) is based on the approach addressed in [4] and it has been redefined to fit the specific requirements of the SCAMP-3 vision system. Firstly, a pre-estimation of the vessel edges is made. Then, both of the main input images needed by PLS (the initial region and the external potential image), are automatically estimated. Finally, PLS evolve to fit the vessel edges.

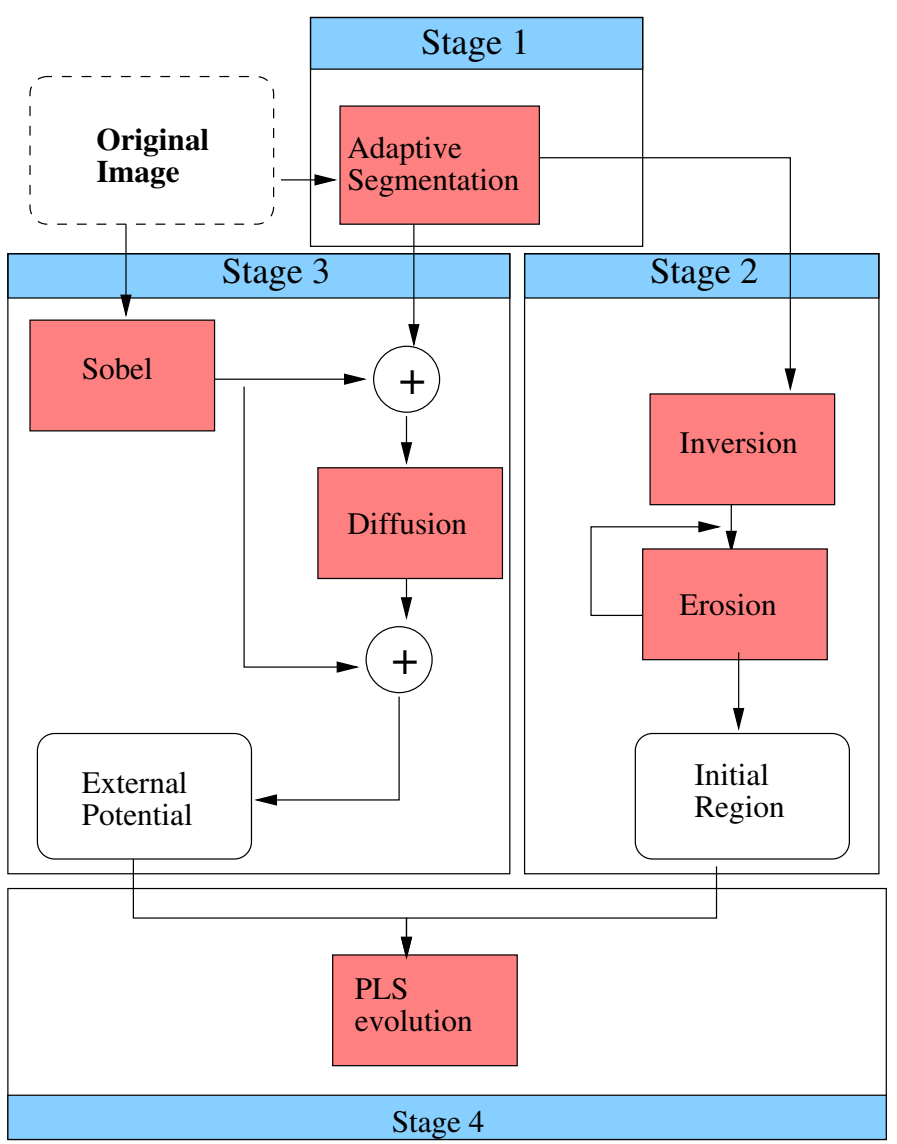

Fig. 1. Flow diagram with the building blocks of the proposed algorithm: Stage 1: Vessel region pre-estimation, Stage 2: Initial region estimation, Stage 3: External potential estimation, Stage 4: PLS evolution

\section{A. Stage 1. Vessel Region Pre-estimation}

The main goal of this stage is to pre-estimate the vessel edges. Due to the non uniformity of the gray level values along the vessels, an adaptive segmentation is needed. Instead of the complex CNN-based approach addressed in [4], another strategy suitable for a hardware implementation has been employed (Fig. 2).

Firstly, the original image is completely blurred by means of a diffusion step. This blurred image contains a local threshold which properly segments not only vessels with a high contrast, but also weak vessels. The blurred image is subtracted from the original one, and finally, the result is binarised based on a fixed threshold value.

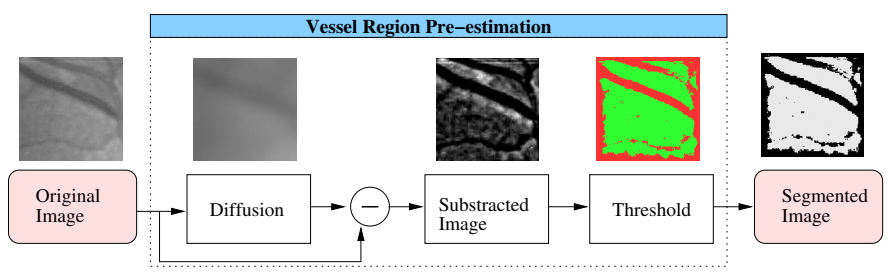

Fig. 2. Stage 1: Vessel region pre-estimation

\section{B. Stage 2. Initial Region Estimation}

The aim of this stage is to obtain a suitable initialisation of the contour locations (Fig. 3).An inversion of the segmented image is initially made to define the regions between the vessels. Since this image can contain vessel discontinuities and regions inside the vessels, it is eroded several times. Thus, the obtained image will contain only regions situated completely outside of the vessel locations. The output of the erosion step will represent the initial condition for PLS.

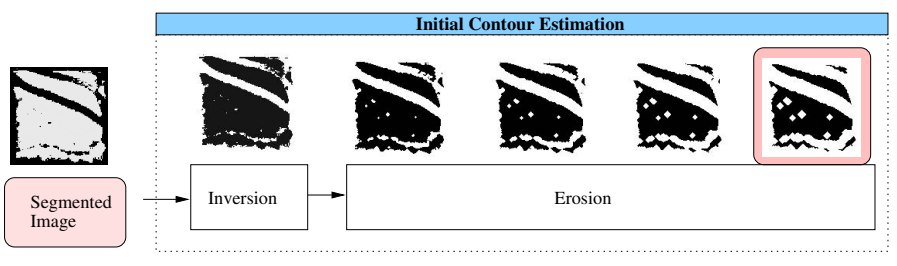

Fig. 3. Stage 2: Initial region estimation

\section{Stage 3. External Potential Estimation}

The external potential image guides PLS towards the exterior of the vessel edges, through a potential field. A sharp potential field valley should be defined to stop the evolution, since PLS can flow through both 1-pixel-width cavities and homogeneous areas even with a high potential field, such as the interior of the vessels. The processing done in this stage is illustrated in Fig. 4.

This stage begins with the application of Sobel operator to the original image, in order to get the actual edges. Although this operator does not introduce much noise, vessel discontinuities appear due to the low contrast of the vessels. The segmented image from the first stage maintains the continuity but at the cost of more segmented noise. The combination of both images gives more robustness to the PLS evolution. It has been implemented following pseudocode: 


\begin{tabular}{l}
\hline \hline$I_{\text {Sobel }}=\operatorname{sobel}\left(I_{\text {orig }}\right)$ \\
$I_{\text {res }}=\frac{3}{4} * I_{\text {Sobel }}+\frac{1}{4} * I_{\text {seg }}$ \\
$I_{\text {diff }}=$ diffusion $\left(I_{\text {res }}\right)$ \\
$I_{\text {extPot }}=\frac{5}{6} * I_{\text {diff }}+\frac{1}{6} * I_{\text {Sobel }}$ \\
\hline \hline
\end{tabular}

where $I_{\text {orig }}$ corresponds to the original image and $I_{\text {seg }}$ is the segmented image from the first stage. A weighted summation is proposed to emphasise the importance of $I_{\text {Sobel }}$, since it contains actual edges, with respect to $I_{\text {seg }}$. Then the result $\left(I_{r e s}\right)$ is diffused $\left(I_{d i f f}\right)$ to obtain a suitable potential field gradients for PLS to flow through. Finally, since a diffusion homogenises the gray level values, specially in the edge locations, the weighted Sobel result is added again to prevent PLS flowing through homogeneous regions and to stop the evolution at the vessel edges.

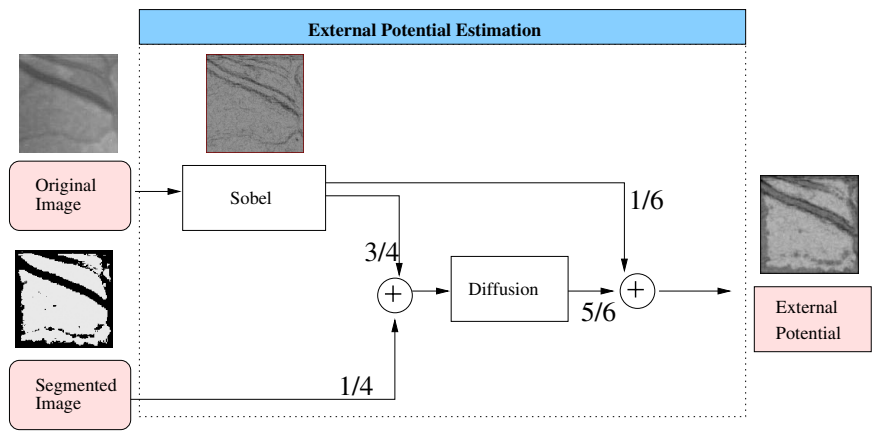

Fig. 4. Stage 3: External potential estimation

\section{Stage 4. PLS Evolution}

PLS evolve to fit the exterior of the vessels using both the initial region and the external potential images, previously computed. The main goal in this stage is to determine the parameters which control the evolution. The internal potential is used to avoid the PLS evolution through vessel discontinuities. Since the vessel edges are situated outside of the initial regions, an inflation potential can help to give more robustness to the evolution. The external potential guides the PLS evolution towards the vessel edges, whereas the internal potential controls the smoothing effect. Due to the specific PLS implementation in the SCAMP system [11], a fixed number of cycles is defined to stop the evolution. Taking into account all these considerations, this stage has been divided into several steps (see Fig. 5).

During the 1st PLS step, the evolution is mainly controlled by means of a high balloon potential, since the external potential is too weak to move the regions. This evolution is performed in this way, since the regions are located far from the vessel edges. Region merging is allowed during this stage, since not only a large region could be split into smaller ones during the 3rd Stage due to the erosion step, but also PLS regions are situated far from the vessel locations. Then, a hole filling operation removes internal regions that appeared due to noise (see Fig. 5). Finally, during the 2nd PLS step, PLS fit the vessel edges relying mainly on the external potential due to the proximity to the vessel edge locations. A smaller
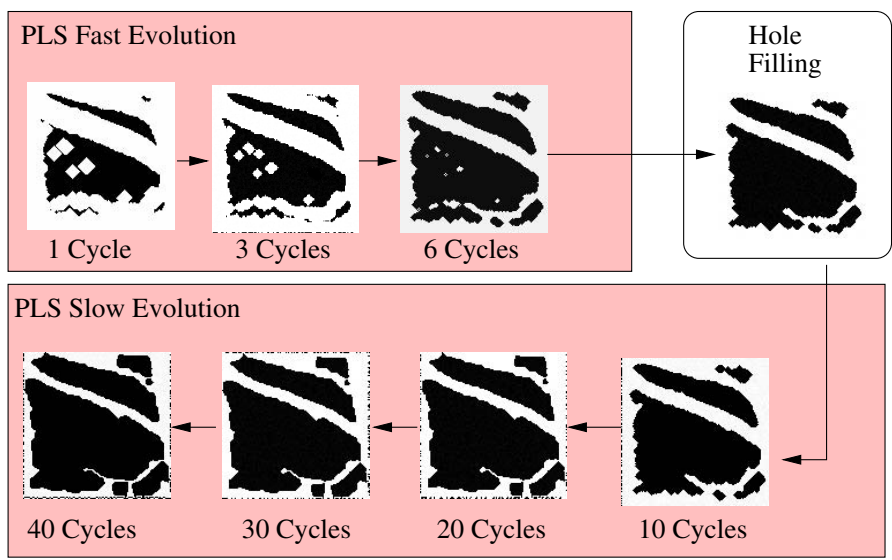

Fig. 5. Stage 4: PLS evolution: 1st PLS Step, Hole Filling, and finally, 2nd PLS Step

balloon potential value is used since the external potential is strong enough to guide the region evolution. In this step, region merging is not allowed in order to maintain the vessel continuity in those cases where neither the external potential nor the internal potential could stop the evolution. The internal potential also controls the smoothing effect of the region, avoiding the PLS flowing through the vessels, maintaining vessel continuity.

\section{RESUlts}

A set of several angiographies has been used to test the implementation with the SCAMP-3 vision system. Each of the angiographies has been split into $128 \times 128$ sub-windows in order to fit the size of the processor array implemented on the chip.

Stage 1 During this stage, the vessel edges are preestimated. The diffusion operation consists of the fast diffusion implemented in the SCAMP-3 vision system, via a "resistive grid" structure. The adaptive segmented results are shown in the 2nd row in Fig. 6. A boundary segmentation effect can be observed; this is due to a zero-padded boundaries in the diffusion operation. A post-processing technique (e.g. a suitable sub-window overlapping) could be used to remove this problem.

Stage 2 The segmented image from the 1st stage, is inverted in order to define the regions between the vessels. These regions are eroded four times to ensure that all the regions are completely outside the vessels (3rd row Fig. 6).

Stage 3 Combining the Sobel result and the segmented image from the 1 st stage, vessel continuity information is maintained in those vessels with low contrast (4th row in Fig. 6).

Stage 4 Using both the initial regions and the external potential images, PLS evolve to fit the actual vessel edges. During the 1st PLS step, a high value potential is used during six cycles. This number of cycles has been selected based on the number of erosion steps used during the Stage 3, which gives an approximation of the distance to the vessel edges. Since the adaptive segmentation gives only a pre-estimation 

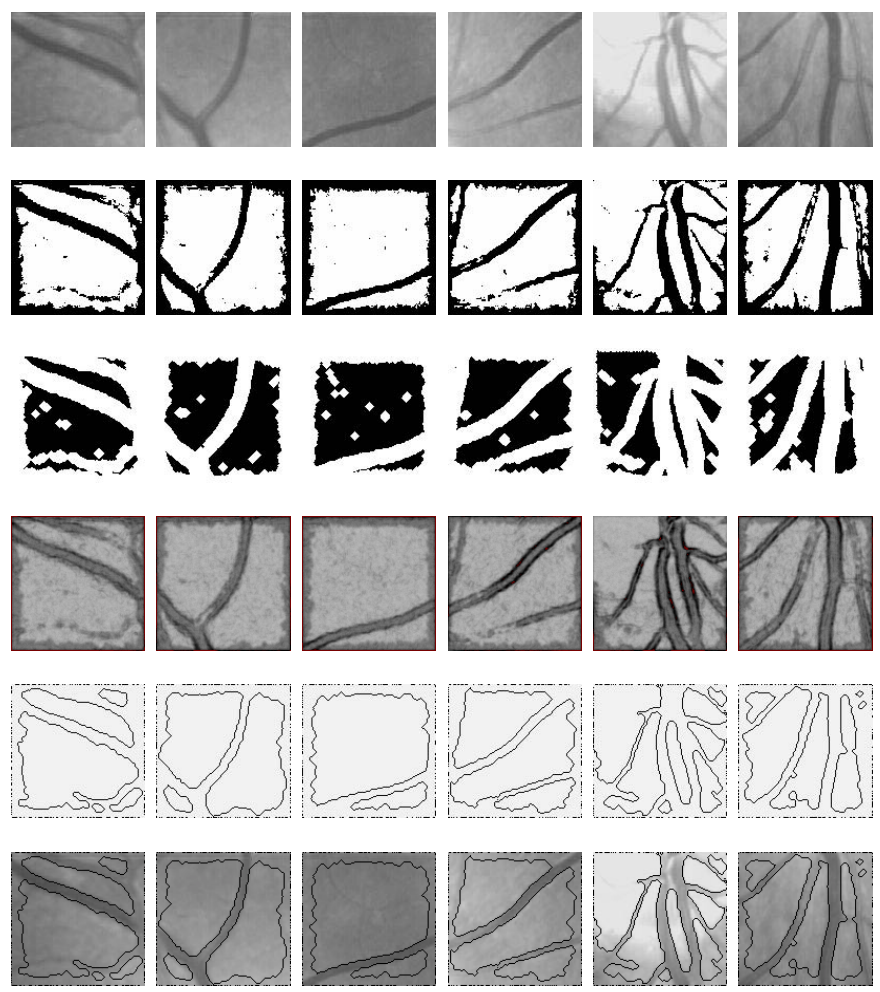

Fig. 6. Output images obtained in each stage: 1st row Original images, 2nd row Pre-estimated vessel region images, 3rd row Initial region images, 4th row External potential images, 5th row PLS output obtained by means of a binary edge detection of the regions, and finally 6th row PLS contours superimposed over the original image

of the vessel edges, the number of cycles used during the 1st PLS step can be increased. Then, the hole filling is applied removing internal regions, and finally, the 2nd PLS step is performed to fit the vessel. The number of cycles during this last step has been empirically established at 40 cycles, since this number of cycles is sufficient in the evolution of PLS for all the sub-windows. Notice the adjustment of PLS to the vessel edges (last row in Fig. 6).

TABLE I

SCAMP EXECUTION TIME FOR EACH STAGE, IN A 128X128 SUB-WINDOW

\begin{tabular}{c|l|c}
\hline \hline No. Stage & Stage & Exec. Time $(\mu \mathbf{s})$ \\
\hline 1 & Vessel Region Pre-estimation & 12.8 \\
2 & Initial Region Estimation & 55.2 \\
3 & External Potential Estimation & 134.4 \\
\hline & 1st PLS Step & 518 \\
4 & Hole Filling & 1954.5 \\
& 2nd PLS Step & 3870.8 \\
\hline \hline
\end{tabular}

One of the goals of the on-chip implementation of the methodology is the measurement of the time performance needed for this algorithm. The computation time required for each stage in the SCAMP-3 vision system is shown in Table IV. The execution time required to perform the whole algorithm for a $128 \times 128$ sub-window is about $6.5 \mathrm{~ms}$. Since the standard angiography size is $640 \times 512$, a total number of 20 sub-windows is required (this number would increase if an overlapping technique is used in order to avoid the noise in the boundaries of the image). So, the global execution time required to process the whole angiography is about $0.13 \mathrm{~s}$.

\section{CONClusion}

In this paper we present an algorithm for the retinal vessel extraction optimised for its implementation on a cellular processor array. The algorithm is based on the methodology addressed in [4], which was developed under the CNN paradigm. However, that proposal could not be implemented in a current generation of CNN chips due to the use of non linear templates for some of the proposed steps.

In this paper, a redefined approach has been proposed in order to implement this methodology in a SIMD cellular processor array architecture. The proposed algorithm has been implemented in terms of linear steps and local operations following the specific requirements of the SCAMP-3 chip. The obtained execution time is much shorter (several orders of magnitude) than conventional PC-based applications.

\section{ACKNOWLEDGEMENT}

The authors would like to thank to Xunta de Galicia, which has partly supported this work (PGIDT04PXIC10501PN and PGIDIT06TIC10502PR).

\section{REFERENCES}

[1] C. Mariño, M. G. Penedo, M. Penas, M. J. Carreira, and F. Gonzalez, "Personal authentication using digital retinal images," Pattern Anal. and Applications, vol. 9, pp. 21-33, 2006.

[2] S. Chaudhuri, S. Chatterjee, N. Katz, M. Nelson, and M. Goldbaum, "Detection of Blood Vessels in Retinal Images using Two-Dimensional Matched Filters," IEEE Trans. Med. Imag., vol. 8, pp. 263-269, 1989.

[3] C. Kirbas and F. Quek, "A review of vessel extraction techniques and algorithms," ACM Comput. Surv., vol. 36, no. 2, pp. 81-121, 2004.

[4] C. Alonso-Montes, D. L. Vilarino, and M. G. Penedo, "CNN-based Automatic Retinal Vascular Tree Extraction," in IEEE Int. Workshop on Cellular Neural Networks and their Applications (CNNA), 2005, pp. 61-64.

[5] L. O. Chua and T. Roska, "The CNN paradigm," IEEE Trans. Circuits Syst., vol. 40, pp. 147-156, 1993.

[6] D. L. Vilariño and C. Rekeczky, "Pixel-Level Snakes on the CNNUM: Algorithm Design, On-Chip Implementation and Applications," Int. Journal of Circuit Theory and Applications, vol. 33, pp. 17-51, 2005.

[7] I. G. Caderno, M. G. Penedo, C. Mariño, M. J. Carreira, F. GomezUlla, and F. Gonzlez, "Automatic Extraction of the Retina AV Index," in LNCS Int. Conf. Image Analysis and Recog. (ICIAR'04), vol. 3212, 2004, pp. 132-140.

[8] T. McInerney and D. Terzopoulos, “Topologically Adaptable Snakes," in Proc. 5th IEEE Int. Conf. Computer Vision, 1995, pp. 840-845.

[9] D. L. Vilariño and C. Rekeczky, "Implementation of a Pixel-Level Snake Algorithm on a CNNUM-based Chip Set Architecture," IEEE Trans. Circuits Syst. I, vol. 51, pp. 885-891, 2004.

[10] P. Dudek and S. J. Carey, "General-purpose 128x128 SIMD processor array with integrated image sensor," Electronics Letters, vol. 42, no. 12, pp. 678-679, 2006.

[11] P. Dudek and D. L. Vilariño, "A Cellular Active Contours Algorithm Based on Region Evolution," in IEEE Int. Workshop on Cellular Neural Networks and their Applications, 2006, pp. 269-274. 